\title{
Modeling of Low Temperature Thermal Networks Using Historical Building Data from District Energy Systems
}

\author{
Ryan Rogers $^{1} \quad$ Vickram Lakhian $^{1}$ Marilyn Lightstone ${ }^{1}$ James S. Cotton $^{1,2}$ \\ ${ }^{1}$ Department of Mechanical Engineering, McMaster University, Canada, \\ ${ }^{2}$ Corresponding Author: cottonjs@mcmaster.ca
}

\begin{abstract}
A Modelica library for modelling and comparing District Energy Systems (DES) and Low Temperature Thermal Networks (LTTN) has been developed. The library consists of six unique models and a series of replaceable sub-models that allow for different scenarios for thermal energy generation. The fluid transport model and losses have been tuned using an empirical data set of a district energy system in operation.
\end{abstract}

An analysis was performed to compare the performance of an existing, operational four-pipe DES against an alternative design that consists of two onepipe LTTN. The results show that the LTTN implementation can drastically reduce the natural gas usage and in turn the carbon emissions of a district energy plant by over $90 \%$ during a two-week period in the transitional month of October for a thermal microgrid in Southern Ontario, Canada.

Keywords: thermal microgrid, district energy, thermal transport systems, carbon emissions

\section{Introduction}

District Energy Systems have historically been implemented in areas with high heating demand and/or cooling to increase energy efficiency due to the utilization of larger industrial generation stations and allow the sharing of energy resources (Lund et al., 2014). These systems use a centralized plant that heats and cools a thermal transport fluid to a set temperature before distributing the conditioned fluid to different buildings within the community using a piping network. Traditionally these piping networks consist of four pipes, a supply and return for heating, and a similar two for cooling. In this way, each building has access to centralized conditioning, and can access energy through an Energy Transfer Station (ETS) that consists of a heat exchanger that interfaces with the district pipes (Figure 1.A).

Although these systems have proven effective at consolidating a community's energy production, especially within large scale institutional campuses, due to increased awareness of the effects of greenhouse gases, there is significant research interest in developing thermal microgrids and a shared thermal economy as a means to reduce emissions.

A thermal microgrid does not depend entirely on this centralized plant, but distributes the load to decentralized energy generation, from traditional units or renewable sources (solar thermal, deep lake cooling, etc. (Li et al, 2016)). The control and balancing of the system is done by an Energy Management Centre (EMC) that allows for better utilization of energy by coupling the energy production resources with demand management strategies. The system can be further exploited for increased efficiency and reduced emissions with the modification of the distribution loop from a high temperature distribution to a Low Temperature Thermal Network (LTTN).

A Low Temperature Thermal Network replaces the two fluid, four-pipe system present in traditional District Energy Systems with a singular low temperature working fluid. Like District Energy Systems, this low temperature fluid is supplied to buildings within a community using a thermal network, which interfaces with each building through an ETS. Although these systems can feature a four-pipe thermal network, traditionally one- or two-pipe thermal networks are used (Bünning et al, 2018). LTTN also differ from the traditional system because they require the use of heat pumps at the building to receive/ inject thermal energy from/into the distribution thermal fluid temperature to match the needs of the internal distribution of the building. This allows for customization within each building as each system is not constrained to match the characteristics of the thermal loop. This necessitates that at every building interface, one or two heat pumps are required to provide the building's heating and cooling demands, depending on whether there is a need for simultaneous heating and cooling.

The implication of this is that the low temperature of the thermal loops allows for a higher level of thermal energy heat capture from sources within the DES or from rejected heat from cooling demand, as the lower temperature within the DES allows for greater temperature ranges for capture, and thus allowing for greater system level energy utilization. Additionally, because the LTTN features a low temperature transport 


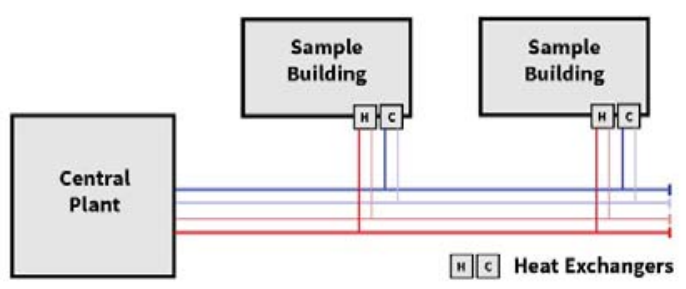

(1.A) District Energy System

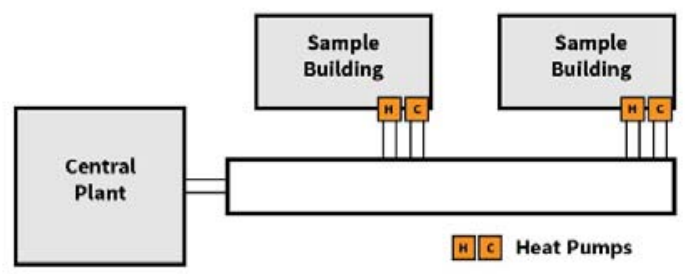

(1.B) One Pipe Low Temperature Thermal Network

Figure 1 Different thermal network systems

fluid, the resulting system energy losses to the environment are reduced due to the lower temperature difference between the piping network and the ground, which results in a lower rate of heat transfer.

A consequence of this LTTN is that each of these heat pumps will have an additional electrical load. However, the countering benefit of LTTN's ability to capture low-grade waste energy from a community provides a potential method to off-set further heating demand. (Lund et al, 2014).

In the LTTN framework (Figure 1.B), the building load is connected to the ETS through a heat pump. The heat pump interacts with the LTTN through a heat exchanger, where both the supply and return connections are connected to the same pipe. This results in a singular pipe which connects all buildings in series and can in turn share energy between buildings. Unlike, the four-pipe District Energy System, after the working fluid leaves the ETS, instead of being returned to the centralized plant, it continues on to the next building. During seasons with simultaneous heating and cooling loads, these series connections allow buildings to act as producers. For example, if a building requires cooling, it will transfer energy into the thermal network, and raise the temperature of the working fluid. This additional energy can be used towards another building's heating demand and, as such, the energy demand upon the centralized plant is reduced. This works similar in reverse, as heating demands cool the temperature of the thermal network and as such benefit other buildings that require cooling. As this pipe is at one temperature, it does not give a preference to either heating or cooling demands of buildings but allows for both. It is the role of the equipment at the central plant to overcome any thermal imbalances and control the setpoint temperature of the thermal fluid.

Due to this tradeoff between higher thermal energy utilization and increased electrical consumption, there exists a need to understand the system dynamics of LTTN systems to determine the impacts on total energy usage and implications on greenhouse gas emissions. Modelica, due to its object-oriented nature and its ease in modeling fluid systems, was chosen to fulfill this need. Using a combination of both existing Modelica equipment models and implementing new analytical models, a modeling hierarchy was created. These models were then tuned using empirical data of an existing traditional four-pipe District Energy Systems before implementing these models as a onepipe LTTN for the same community, as is described in Section 2. The performance of both these systems was compared by contrasting total resource utilization, peak electrical power requirements and carbon emissions of both systems.

\section{Models}

In order to accurately model both District Energy Systems and LTTN, a Modelica library was created to model the piping, ETSs, and a simplified EMC within the system. These models incorporate components from both the base Modelica Standard Library as well as the AixLib Building systems library (Müller et al., 2016).

\subsection{Thermal Pipe Loss Model}

The thermal pipe loss model, DistrictPipe is utilized to simulate the heat losses from a buried pipe to the environment. The model is based on an analytical model for steady state pipe losses developed by Wallentén (1991). The model considers pipe diameter, insulation diameter, buried depth and ground surface temperature taken from climate data. It calculates a heat transfer resistance between the working fluid and the ground using the buried depth, pipe size, and the thermal conductivity properties for the ground and pipe (1). Using this resistance, the temperature between the fluid and the ground surface can then be used to calculate the energy lost across the pipe.

$$
q=2 \pi \lambda_{g}\left(T_{1}-T_{o}\right) \cdot h_{1}\left(H, r_{o}, \beta\right)
$$

Where $q$ is the pipe heat losses

$\lambda_{g}$ is the thermal conductivity of the ground $T_{1}$ is the temperature of the fluid in the pipe $T_{0}$ is the temperature at the ground surface $h_{1}$ is the heat transfer coefficient for the losses $H$ is the buried pipe depth

$r_{o}$ is the outer radius of the pipe insulation $\beta$ is a dimensionless parameter relating the ground's thermal conductivity to that of the pipe's insulation. 


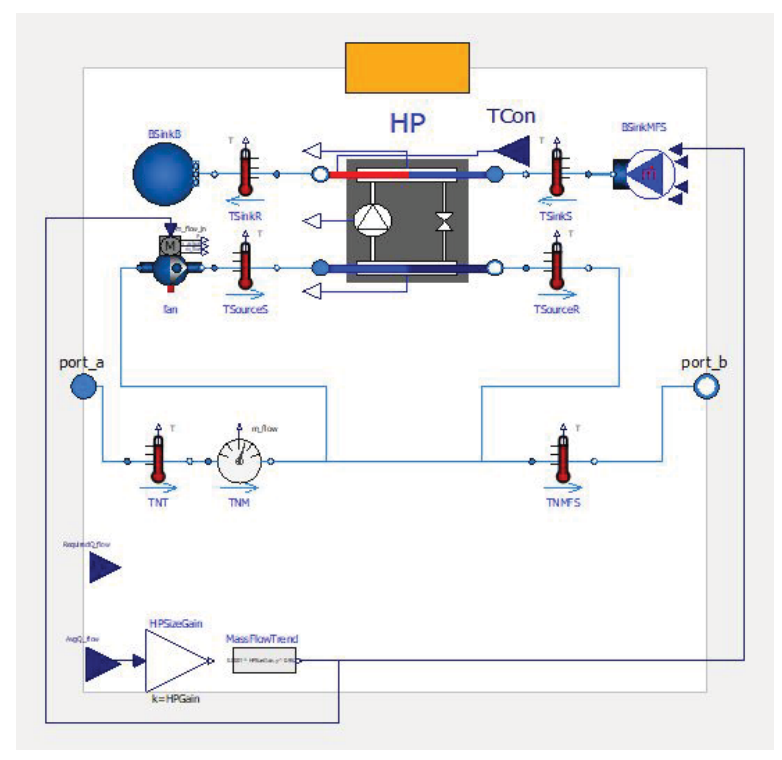

Figure 2. One-Pipe Energy Transfer Station model

Using the Modelica standard library, the resistance and temperature parameters were integrated in the DynamicPipe model which then created a discretized heat transfer model for the system. The DynamicPipe model also calculates the pipe pressure losses for the DistrictPipe model allowing comparisons to be made between different pipe network configurations pump power requirements.

\subsection{Four-Pipe Energy Transfer Station}

The four-pipe ETS is a standard energy transfer station that consists of a pump, heat exchanger with unity efficiency, and a return outlet. The pump is used to direct a set amount of working fluid away from the main header and into the heat exchanger. This diverted mass flow set point is calculated using a relationship derived from equipment datasheets that relates average buildings heating or cooling requirements to a heat exchanger's nominal mass flow rate.

The building load interfaces with the ETS through two Modelica RealInput interfaces. The first connector supplies the seasonal average energy requirements for the building which is used to calculate the mass flow rate to the heat exchanger. The second is the energy required by the building from the ETS. This is used to dynamically calculate the inlet temperature to the heat exchanger on the building side, changing the heat exchangers performance and describing the load within the building itself. A simple control strategy based on a building set temperature and equipment trends gathered from datasheets is also implemented which controls the energy transfer, as well as enforces maximum and minimum energy draw constraints on the heat exchanger.

\subsection{One-Pipe Energy Transfer Station}

The one-pipe ETS expands on the four-pipe ETS by replacing the heat exchanger with a heat pump model (Figure 2). Since the thermal output of this model is equipment dependent, two models have been made, one for heating and one for cooling. In both cases, Carnot based heat pumps were used from AixLib (Müller et al, 2016). These models condition the working fluid to a fixed outlet temperature and calculate the Coefficients of Performance (COP) and power consumption for the equipment based on a Carnot efficiency.

$$
\text { COP }=\eta_{\text {Carnot }} \frac{T_{\text {hot }}-T_{\text {cold }}}{T_{\text {hot }}}
$$

Where $T_{\text {hot }}$ is the temperature of the condenser $T_{\text {cold }}$ is the temperature of the evaporator $\eta_{\text {Carnot }}$ is the Carnot efficiency of the heat pump when compared to the ideal cycle

Although this model is not fully based on standard equipment, the Carnot models allow for a range of buildings to be tested without the reliance on equipment look up tables. To further improve the accuracy of the models, a building set temperature control system as well as minimum and maximum energy draw constraints were enforced on the heat pump. These constraints ensured that the COP operates within a reasonable range of existing commercial equipment $(\mathrm{COP}=[3.09-4.20])$.

\subsection{Energy Management Centre}

The Energy Management Centre model simulates the centralized plant production for both the District Energy System and the LTTN. It contains the fluid conditioning system, sensory models used to determine the total energy draw and an equipment dispatcher for the energy production. This dispatcher connects to four replaceable sub-models. These models break down the energy production into four categories: on-peak heating (x hours/day), off-peak heating (y hours/day), on-peak cooling ( $\mathrm{z}$ hours/day) and off-peak cooling (w hours/day), where peak refers to the hours within a day when the equipment would be operated during the hours when there is electrical peak demand upon the electrical grid. This is of interest in areas where baseload electricity production is primarily emissions free, except for peak usage where there is fossil fuel generation (e.g. Ontario or British Columbia, Canada). This typically refers to the between the hours of 7:00 to 19:00 per day during the month of October. 


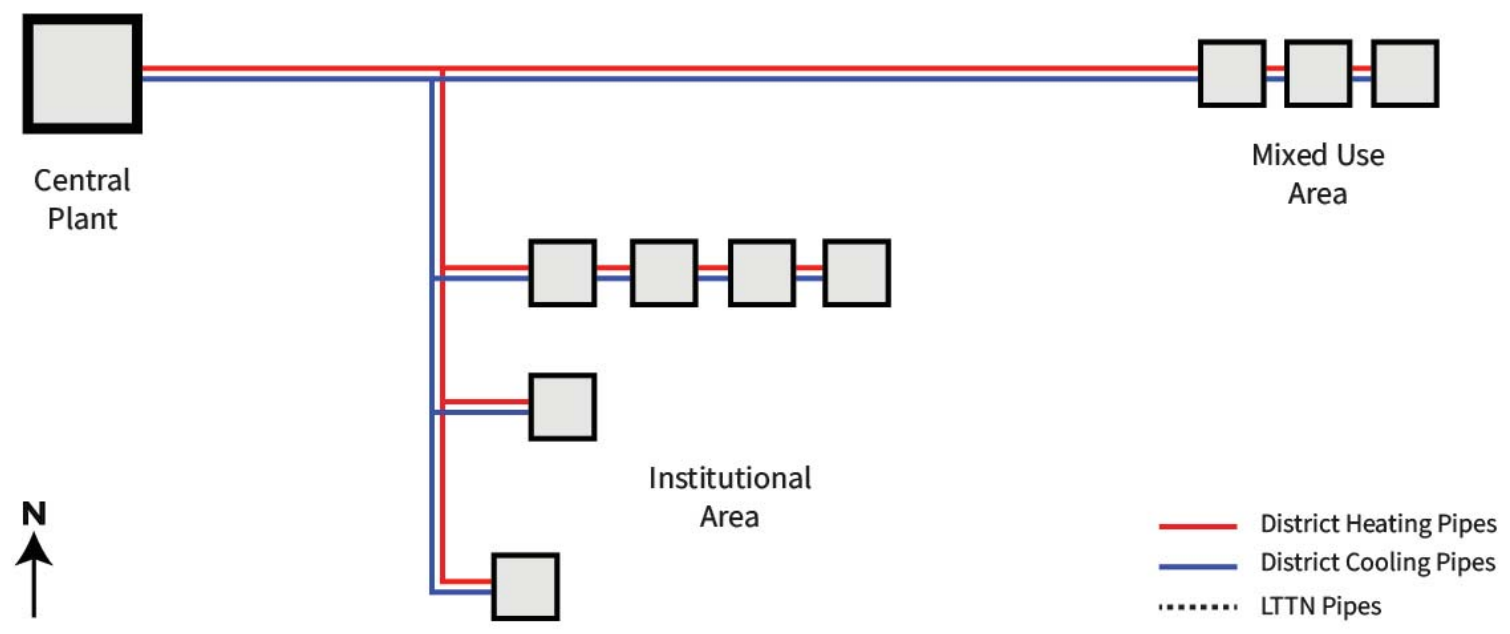

Figure 3. District Energy System case study

These components within the model can easily be replaced with four different models of mechanical equipment: a boiler, a combined heating and power plant (CHP), an air source electric chiller and a ground source heat pump. By implementing these models within the four different energy production categories, they can convert the EMC's thermal energy production requirements to electrical power requirements, natural gas demand and carbon dioxide emissions which can then be used for system comparisons.

\subsection{Supplemental Models}

Additionally, various sub-models were also created to simulate the thermal network system. One of these sub models is a building load model, that incorporates real building data within the Modelica environment through a timetable. This system can handle data at a variety of time steps and also produces the buildings average energy requirements to the other models.

Another sub-model that was created, the IsoPipe model, which was used to model the heat energy and pressure losses for district energy pipes that were not buried underground. These could exist within the interior of buildings or within underground civil infrastructure such as parking garages or utility corridors.

Additional to these components, an array of connectors was also made in order to reduce the total number of connections required within the high-level thermal network models.

\section{District Energy Case Study}

To validate the four thermal energy loop models, a case study was chosen for simulation. This case study focuses on a nine-building district energy system located in a high-density community within Southern Ontario, Canada. The community contains mixed use, institutional, commercial and residential building types. Figure 3 shows the general layout of this system; the total foot print of the community is approximately $0.40 \mathrm{~km}^{2}$.

\subsection{Design and Layout}

The District Energy System can be portioned into two major sections. The first area is the mixed-use area located on the east end of the community. These three buildings contain both office space and residential apartments all which require both heating and cooling from the district system.

The second area located in the southern section of the community consists of six institutional buildings. The four buildings located to the north, consist of an office building and three community institutional buildings. Additional to this, a large municipal building is located in the southernmost part of the campus while a medical research facility is centrally located.

Both areas are connected to the centralized plant using a thermal network. The heating pipes are 15.24 $\mathrm{cm}$ diameter steel pipes with an insulated diameter of $24.51 \mathrm{~cm}$. While the cooling pipes are larger diameter $20.32 \mathrm{~cm}$ HDPE without insulation.

\subsection{Comparison between Model and Observations}

Real-world observations were used to tune the model to more closely match reality. To ensure the model recreated the existing system as closely as possible, information was obtained for the existing physical four-pipe system. 


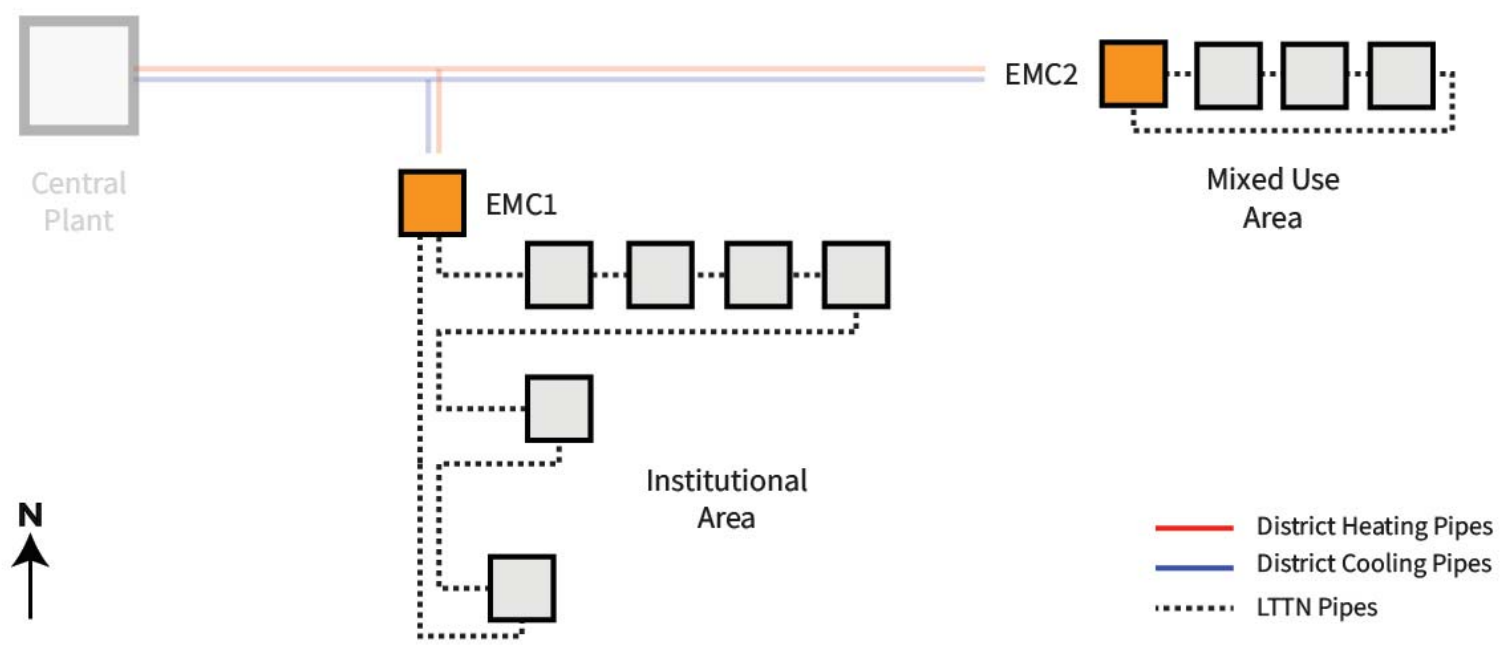

Figure 4. Low Temperature Thermal Network implementation

This included all pipe lengths, equipment efficiencies and the building load data within the system. This data, captured at five-minute intervals, was then used to reconstruct the physical plant as a Modelica model and comparisons were then made between EMC performance of the simulated model and the physical plant.

The existing system observes a $24 \%$ loss in energy from the total energy generation of the system at the central plant, to where the energy is delivered to the building loads. The system was simulated in Modelica with the library described in Section 2. This indicated that approximately half of the total losses $(13 \%)$ were heat loss from the buried pipes to the environment, using expected values for the thermal conductivity of the soil and heat exchanger efficiencies. Both the thermal conductivity of the soil and the heat exchanger efficiency were tuned to assess the sensitivity of the error. This showed that while step changes in ground thermal conductivity had little effect on the pipe losses, the heat exchanger efficiencies could potentially account for the error. This led to the decision that to best address the remaining $11 \%$ error more experimental work is needed to determine the true nature of these losses before the library can be better tuned.

\section{LTTN Implementation}

To showcase an example of a LTTN implementation, the same case study that was used to validate the fourpipe District Energy System was decomposed into two communities that were then modeled with two, onepipe LTTNs (Figure 4).

\subsection{Design and Layout}

In this layout, the two distinct areas outlined with the District Energy System have been separated and outfitted with their own LTTN systems. Both of these systems consist of individual one-pipe networks. For this example, the distribution pipe was modelled using the insulated pipe present in the physical system (15.24 $\mathrm{cm}$ pipe diameter, $24.51 \mathrm{~cm}$ insulation diameter). Thermal losses to the environment were taken into account, as with the observed data for the four pipe District Energy case.

\section{Results and Discussion}

The results from operating the two different systems for a two-week period in October of 2017 are discussed in this section. It should be noted that there are simultaneous demands for heating and cooling in this month, typical of October in Southern Ontario, Canada. For this comparison, the pipe specifications outlined in the previous sections were used. For both the District Energy System and the LTTN, the heating production was supplied using a boiler and cooling production was supplied with a chiller regardless of peak timing. For the two different areas in the LTTN, the average thermal energy requirements throughout the two-week period are outlined in Figure 5 with the total community energy requirements equal to approximately $52.96 \mathrm{MWh}$ for the period. Both

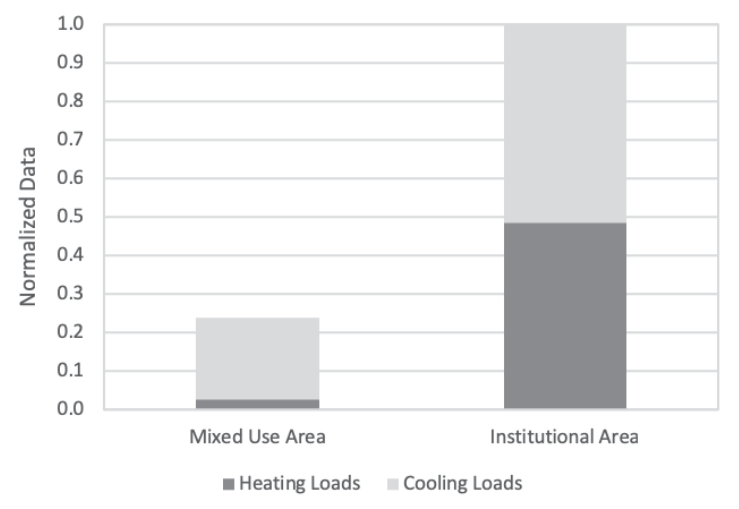

Figure 5. Energy demand distrubution for the case study community 


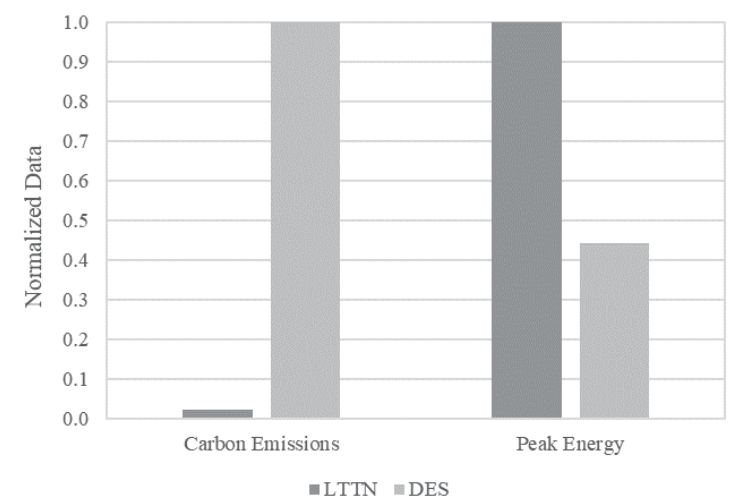

Figure 6. Carbon emission and peak power comparisions

systems were then compared based on total power consumption, total electrical costs and carbon emissions.

\subsection{Total Energy Usage:}

\subsubsection{Peak Electricity Usage}

The total peak electrical power use for this two-week period in October with the traditional four-pipe District Energy system is $5.8 \mathrm{MWh}$, while in the case of the LTTN it is 13.0 MWh (Figure 6). This is due to the additional heat pump utilization with the LTTN.

\subsubsection{Total Resource Usage}

The four-pipe system uses 1.2 MWh of energy in the form of electricity for pumping and requires $9.2 \mathrm{MWh}$ of electricity for cooling. Additionally, it also uses 23.0 MWh of thermal energy in the form of natural gas for heating at the centralized plant.

Comparatively, the LTTN requires $0.7 \mathrm{MWh}$ of electricity for pumping, 5.4 MWh of electricity for cooling and $0.8 \mathrm{MWh}$ of natural gas energy for heating. Additionally, the system utilized 15.1 MWh of electricity for the heat pump operations, however this additional electrical energy made the system more efficient and resulted in a 34\% total energy reduction when compared to the District Energy System.

Figure 7 and Figure 8 show the dynamic energy generation of both the DES and LTTN system respectively. Although the LTTN utilizes 34\% less energy, it still has high generation periods equivalent to that of the DES system during periods where cooling is dominate. This $34 \%$ decrease in energy is a direct result of the energy sharing which is taking place between capturing rejected heat from cooling loads, and conversely for heating loads.

Additionally, the LTTN model also exhibited much lower pipe losses than the DES model. Using the same insulated piping geometry for both cases, the lower temperature set point of the $\operatorname{LTTN}\left(25^{\circ} \mathrm{C}\right)$ led to a $95 \%$ reduction in pipe losses when compared to the higher DES set point $\left(75^{\circ} \mathrm{C}\right)$.

Figure 7 also gives insight into the nature of the mixed loads within this DES. Although the community has almost equivalent heating and cooling generation requirements, these generation periods are offset by a standard 12-hour period.

\subsection{Carbon Dioxide Emissions}

Considering the carbon dioxide emissions, it was found during the transitional month of October, due to a mix of heating and cooling demands (Figure 5), the LTTN had much lower carbon emissions than the traditional District Energy System (Figure 6). This stems from the heat pumps ability to reduce total centralized plant production by capturing rejected thermal energy from periods that require both heating and cooling. During periods of heating demand, the heat pumps within the LTTN provide heating and in return cool the thermal network. Similarly, during periods of cooling demand, the heat pumps provide cooling and in turn heat the thermal network. This rejected energy coupling reduces the total production at the centralized plant which results in lower emissions from natural gas

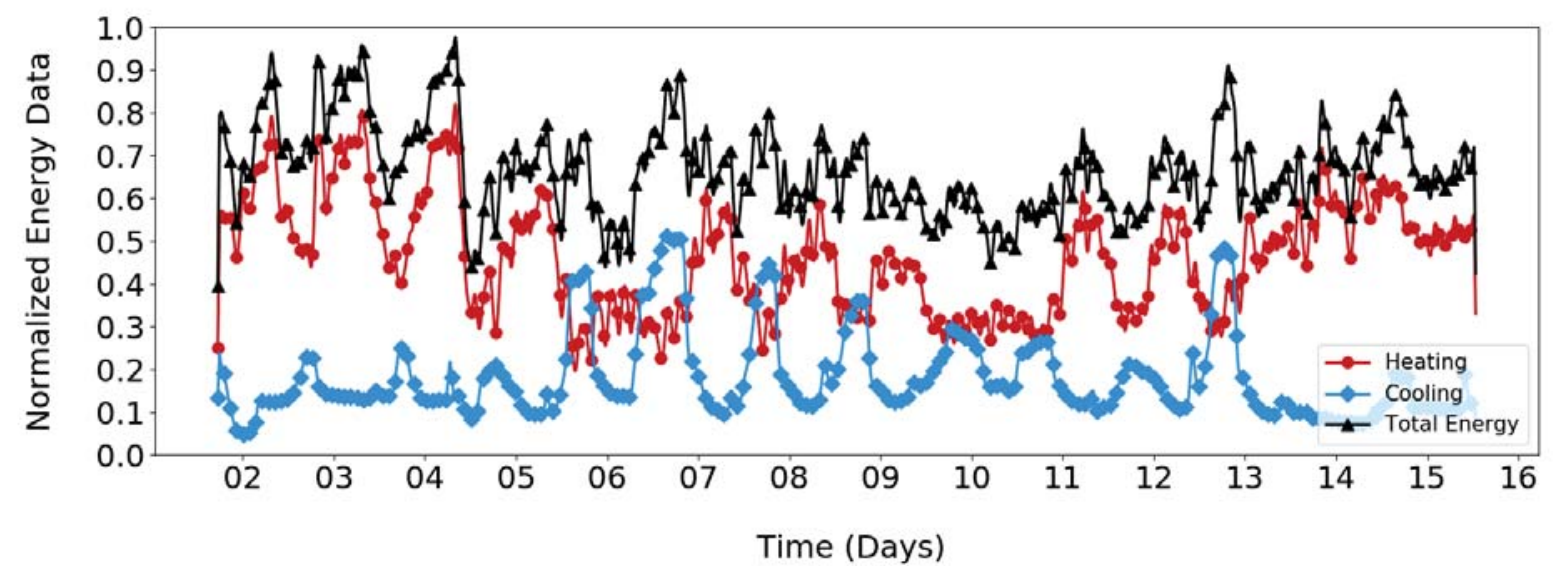

Figure 7. DES Energy Generation Comparison: Heating (Natural Gas Heating Load), Cooling (Electrical Load) 


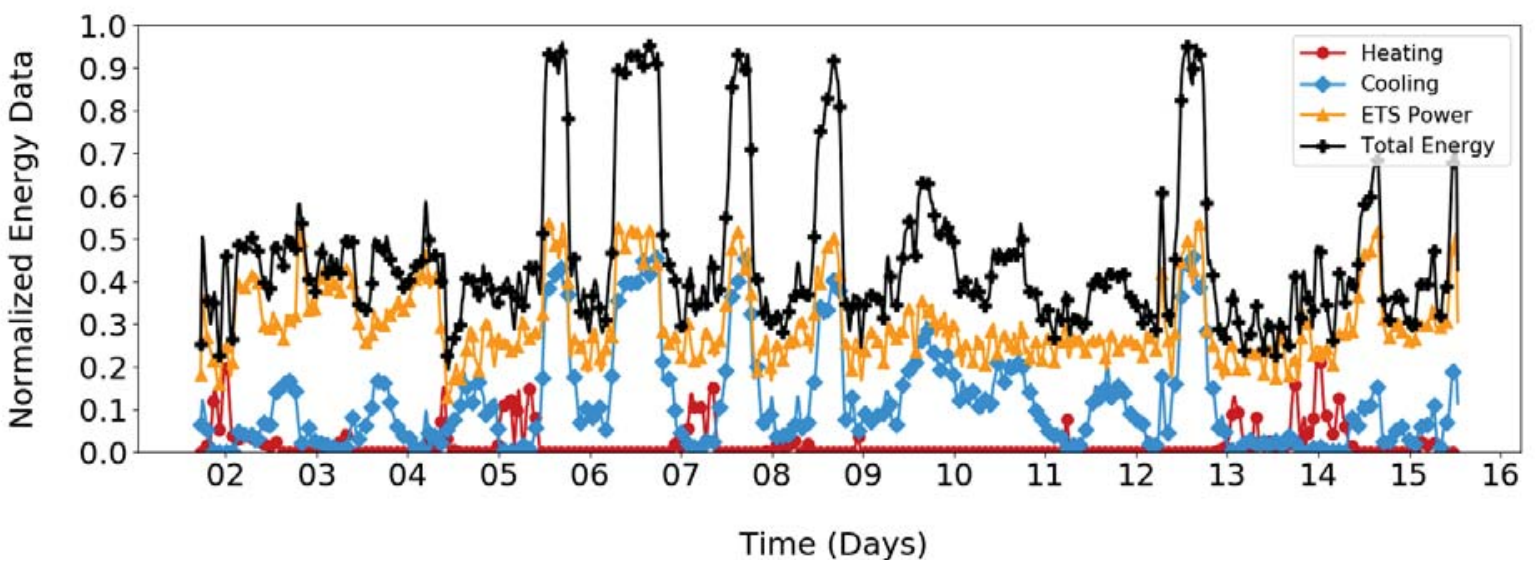

Figure 8. LTTN Energy Generation Comparison: Heating (Natural Gas Heating Load), Cooling (Electrical Load), ETS Power (Electrical Load)

heating. This reduction remains true even when accounting for the additional carbon dioxide production from using heat pumps when there is fossil fuel generation on the electrical grid during peak times. During these peak periods the impact of the waste energy recovery benefit still reduces carbon emissions by at least $56 \%$ based on calculations from Environment Canada (2017) (51 kg CO $/ \mathrm{Gj})$.

\section{Conclusions}

A library for modelling Low Temperature Thermal Networks has been developed to help characterize the performance of these new energy systems. The library consists of six unique models for the simulation of both traditional four-pipe District Energy Systems and onepipe LTTNs. The library models were used to simulate an existing four-pipe District Energy System which provided real energy data for both energy demand and generation for a case community. This comparison indicated an $11 \%$ error between the predicted values and the historical values of the thermal losses in the distribution grid, an error that is being addressed through additional experimental analysis.

Beyond this comparison, a LTTN design was then implemented in lieu of an existing District Energy System in a nine building, high density community to demonstrate the effectiveness of the LTTN. By simulating the LTTN, it was found that although the single pipe design's integration of heat pumps increases the peak electrical power requirements of the community by $7.2 \mathrm{MWh}$, the waste energy recovery potential of the system can reduce the carbon emissions for the system by at least $56 \%$. Additionally, the system reduced the total energy utilization by $34 \%$ by utilizing electrical energy to improve the efficiency of the heating and cooling process.

\section{Future Work}

To further improve the Modelica library presented, some revisions are still necessary to account for the additional $11 \%$ energy losses in the validation case. These steps include experimentally testing the DES system to determine the nature of these losses and then adjusting the Modelica library to account for them.

\section{Acknowledgements}

Natural Sciences and Engineering Research Council of Canada, Ontario Centres of Excellence and the team from HCE Energy Inc., Shawn Forbes, Ankur Mehrotra and Savan Trivedi.

\section{References}

Felix Bünning, Michael Wetter, Marcus Fuchs, and Dirk Müller. Bidirectional low temperature district energy systems with agent-based control: Performance comparison and operation optimization. Applied Energy, 209, pp. 502-515, 2018.

Mengyu Li, Xiongwen Zhang, Guojun Li, and Chaoyang Jiang. A feasibility study of microgrids for reducing energy use and GHG emissions in an industrial application. Applied Energy, 176, pp. 138-148, 2016.

Henrik Lund, Sven Werner, Robin Wiltshire, Svend Svendsen, Jan Eric Thorsen, Frede Hvelplund, and Brian Vad Mathiesen. 4th Generation District Heating (4GDH) Integrating smart thermal grids into future sustainable energy systems. Energy, 68, pp. 1-11, 2014.

D. Müller, M. Lauster, A. Constantin, M. Fuchs, and P. Remmen. AixLib - An Open-Source Modelica Library within the IEA-EBC Annex 60 Framework. BauSIM, pp. 3-9, 2016.

P. Wallentén. Steady-state heat loss from insulated pipes. Byggnadsfysik LTH, Lunds Tekniska Högskola, pp. 13-15, 1991.

Environment Canada, Greenhouse Gas Sources and Sinks in Canada Part 2: Canada's Submission to the United Nations Framework Convention on Climate Change. National Inventory Report 1990-2015, 2017. 
Modeling of Low Temperature Thermal Networks Using Historical Building Data from District Energy Systems 